\title{
Simulation and Implementation of Microcontroller Based Printed Circuit Board Ready Circuits for Technical Training and Demonstration
}

\author{
Blessed Olalekan Oyebola ${ }^{1}$ and E. Eze Blessing ${ }^{2}$ \\ ${ }^{1}$ Department of Computer Engineering Technology, Gateway (ICT) Polytechnic Saapade, Nigeria \\ ${ }^{2}$ Department of Computer Engineering, The Federal Polytechnic, Ilaro, Nigeria \\ E-Mail: blessedolalekan@gmail.com, ebereblessing247@gmail.com
}

\begin{abstract}
The paper presents designed and constructed microcontroller based PCB ready on five automation circuits as an attempt to aid technical education on microcontroller circuits' simulation in Computer Engineering Technology. The designs were of three stages that include circuit design and simulation by using Proteus software, printed circuit board (PCB) production using manual techniques and construction of five selected circuits (Seven Segment to Display Number 0-9, seven Segment Counter to Count 0-99, Matrix Led to Display A-Z, Digital Thermometer and Motion Sensor to Switch 10 Points of Loads) for purpose of training. The microcontroller programing were done in Mikro $\mathrm{C}$ programming platform. The presented hand-on circuits have proved to be instructive to trainees.
\end{abstract}

Keywords: Load, Matrix, Microcontroller, Motion, Printed, Segment, Simulation

\section{INTRODUCTION}

Printed circuit board is an essential part of electronics. Alternately, the acronym has also accounted for printed wiring boards and printed wiring cards, which are essentially the same thing (1). Due to the crucial role of these boards in everything from computers to calculators, PC board material selection should be undertaken with care and knowledge for electrical necessities of a given piece of equipment (2). Before the development of the PCB, circuit board materials were mostly covered by nests of entangled, overlapping wires that could easily fail at certain junctures (3). They could also short circuit once age took hold and certain wires started to crack. As could be expected, the manual process that went into the wiring of these early boards was confusing and painstaking (4). As an increasing variety of everyday electronic components began to rely on circuit boards, the race was on to develop simpler, more compact alternatives, and this led to the development of the material, Printed Circuit Board (PCB). With PCB materials, circuits can be routed between hosts of different components. The metal that facilitates the transfer of current between the board and any attached components is known as solder, which also serves a dual purpose with its adhesive qualities (5). This paper presents design and construct a PCB ready using micro-controller on the following circuits: Seven segment to display 0-9, Seven segment counter to count 0-99, Matrix led to display A-Z, Digital thermometer, LM 35 and Ten points of light controlled by motion sensor.
This paper will serve as a template or sample for teaching beginners in Electronics how to construct and program electronic circuits in schools and can be used for demonstrations to students during Electronic practical. During the completion of this paper, a lot of knowledge had been applied from the Electronics practical studied from early semester until the end of semester. Basically this paper focused on:

1. Identifying the Components and Materials: In the process of identifying the components and materials going to use, the components and materials related in this paper were made ready in the stock.

2. Designing and Layout: In this process, Proteus software, where it is providing the designing, simulation and layout design for PCB.

3. Testing and Analyzing: In this process of testing and analyzing, the job related at the lab had been performed. The testing part has to test using the multimeter while the analyzing processes have to do at the lab.

\section{RELATED WORK}

Simulation has long been one of the most important steps in the design of integrated circuits. When it is not possible to probe most points in the circuit and when mistakes can cost hundreds of thousands of dollars, simulation software can prove invaluable (6). But when it comes to printed-circuit boards many designers are reluctant to use simulation software. As a result, they are missing out on substantial advantages, which include better circuit performance and reliability, improved documentation, and deeper understanding of circuit operation (7).

\section{A. PCB Timeline}

Before printed circuits became the common component used in electronics, point to point construction was used. This meant some extremely bulky and unreliable designs that required large sockets and regular replacement. Most of these issues were directly addressed when PCBs went into regular production. 
1920 - The early PCB material could be almost anything, from Bakelite and Masonite to plain old thin pieces of wood. Holes could be drilled into the material and flat brass wires would be riveted onto it. It may not have been pretty, but the concept was there, and it worked. It was often used in radios and gramophones at the time.

1947 - First double sided PCB's with plated through holes produced.

1950 to1960 - The types of materials used for the board was shifting to different resins and other materials, but they could still only be printed on a single side. The wiring would be printed on one side and the electrical components would be on the other. Still, it was a much more efficient option than bulky wiring, so it was starting to see a wider adoption.

One of the biggest steps forward came in 1956 when the U.S. Patent Office granted a patent to a small group of scientists representing the U.S. Army for the "Process of Assembling Electrical Circuits.” At the time, the process involved drawing the wiring pattern and then photographing it onto a zinc plate. This plate could then be used to create a printing plate for an offset printing press. This is what was used to print the wire in acid resistant ink on the copper foil, which could then be etched by an acid solution.

1957 - IPC (The Institute of Printed Circuits) formed and hold first meeting in Chicago IL (8).

1960 - Multilayer (4+ layer count) PCB’s begin production.

1960 to 1970 's- Boards were designed using 4:1, red-andblue line vellum method for hand-taping components and tracks. A precision camera then produced the 1:1 negative manufacturing film. An experienced designer could layout and tape a board at the rate of about two hours for each equivalent 14-pin IC on the board.

1970 t0 1980 - The circuitry and overall size of the boards were starting to get a lot smaller by the 70s, and hot air soldering methods began to be used. This is also when the Japanese developers began to create screen processes that used various aqueous developed LPIs (liquid photo image able masks). This became the industry standard over the years. Gerber Scientific introduces RS-274-D as a machinebased format for vector photo plotters.

1980s - Surface mount parts became the preferred option over through-hole components, which led to further size reductions while maintaining the same level of functionality.

1986 - RS-274X released as an enhancement to RS-274-D data format. New version supports embedded aperture information relieving the need for external aperture definition files.
1992 - Valor Computerized Systems founded introducing Genesis 2000 software for PCB CAM and DFM.

1990s - While the complexity of modern circuit boards continues to go up, the size of the boards and costs of materials has generally been able to go down. Once developers were able to start using multi-layer circuit boards they were able to minimize the size and incorporate combinations of rigid and flexible PCBs in a range of devices. Going forward, new developments will continue to produce more efficient circuits that can effectively meet the needs of rapidly growing technology.

1995 - US PCB production reaches $\$ 7.1$ billion, topping $\$ 7$ billion for first time.

1995 - Use of micro-via technology in PCB production starts, ushering in the era of HDI (High Density Interconnect) PCB's.

1997 - Valor Computerized Systems releases ODB ++ printed circuit board manufacturing data format into public domain.

2000 - US PCB fabrication market peaks at more than $\$ 10$ billion.

2000s - PCB Real Estate becomes even tighter with 5-6mil trace \& space becoming commonplace. Hi-tech shops fabricating boards with 3.5 to 4.5 mil trace \& space in production quantities. Flex and Rigid-Flex PCBs has become an affordable option and is widely used.

2010s - ELIC (Every Layer Interconnect) production begins.

PCBs have copper tracks to connect the holes where the various components are located. They are specially designed for each and every circuit and build construction very easy. Though, making the PCB necessitates special tools. The different types of printed circuit boards mainly include the following (8) : Single Sided PCBs, Double Sided PCBs, Multilayer PCBs, Rigid PCBs, Flex PCBs and Rigid-Flex PCBs

\section{A. The Importance of Printed Circuit Boards}

Printed Circuit Boards (PCB) are thin plates which are usually made of fiberglass, laminated materials, or composite epoxy used as physical base to support chips and electronic components. These boards are printed or etched with conductive pathways to form circuits that will power electronic devices or gadgets. They support different electronic components like integrated circuits, resistors, and transistors, which are interconnected in the copper tracks. These components are put by drilling holes in the board and soldering them into the circuit pattern (9). 
Printed circuit boards are the motherboards of any electronic device from simple beepers, phones to computer systems, radars, and state of the art communication technology. They are self-contained modules that accommodate elements to form electrical circuit in order to activate electronic devices. Most televisions, cellular phones, radios, tablets and digital cameras have one or more PCB's. Here are some of the reasons why PCBs are essential to electronics and technology industries:

1. They give mechanical support to electronic components.

2. They connect different components to others essential materials.

3. They give input/output connections that make devices functional.

4. They provide electrical impedance matching or the practice of designing opposition of the signal source to minimize or maximize the transfer of power.

5. They serve as conductors of heat transfer.

6. They provide electromagnetic shielding to guard the electronic components and cables
These gave rise to a three-part methodology in order to create quality boards. These three steps are schematic capture, simulation, and final layout. Schematic capture is creating design, picking the essential components, and placing them to the scheme. The electrical interconnection is then checked to ensure the board's capacity. Simulation tools manipulate the model scheme to check the magnetics, signal integrity, and physics. Final layout is the final phase and with the use of internet, the board design can be changed according to real-world specifications that make data accessible anytime and anywhere. This ensures that evaluation and re-evaluation can be done easily before the actual manufacturing process (9).

\section{DESIGN AND CONSTRUCTION}

This section describes analyses and gives detail explanation on how components used in this paper are connected and also explore details characteristics of each of them.

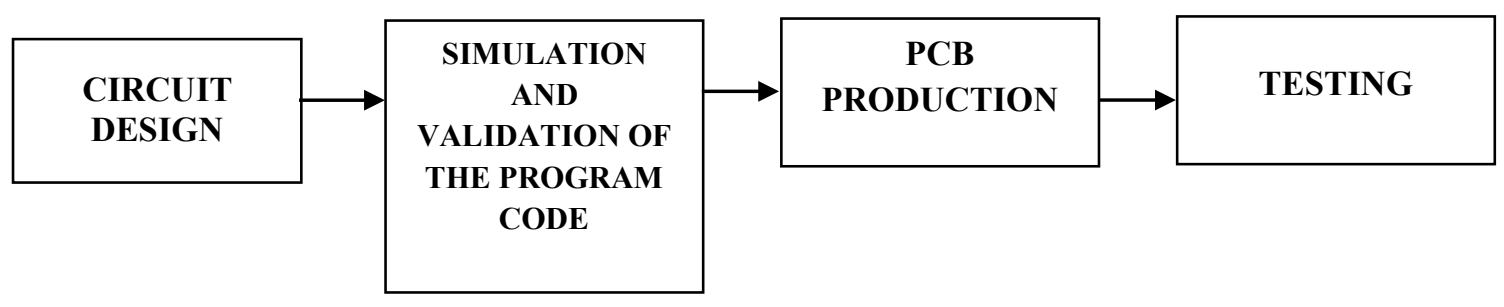

Fig. 1 Project Block Diagram

1. Circuit Design: The circuit design section is when the idea of the circuit is conceived and drafted/designed on a sheet of paper.

2. Simulation and Validation of the Program Code: This section is where the drafted circuit is simulated on simulation software and the code is written and any possible corrections are made before the prototype of the simulated circuit is produced.

3. PCB Production: After the simulation of the circuit is made, the PCB is simulated and produced on a copper coated sheet.

4. Testing: This is where the circuit is tested.

\section{A. Software Development}

For the programming codes see Appendix A. Diagram of the Flow-Chart is shown on Figure 14.
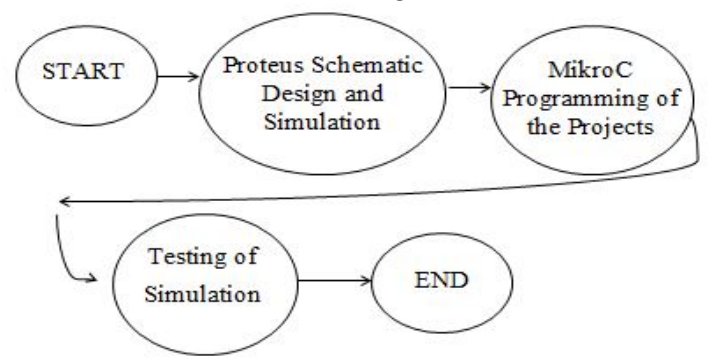

Fig. 2 Flow-Chart of the Project

\section{Software Algorithm}

1. The 8051 microcontroller interfaces the major components in the circuit paper.

2. In the oscillatory circuit of the microcontroller, two 33pf capacitors were used to store little amount of charges so it could function well.

3. The LCD (Liquid Crystal Display) has 16 legs and the major legs that are working is just 12 . The Mikro c programmer was used to program it; the V of the LCD is connected to the potentiometer. The LCD is used for graphical display of what is happening between the microcontroller and the reader.

4. Potentiometer: The potentiometer is connected to the LCD; it helps in working for the contrast of the LCD.

5. The resistor connected to the MCLR of the microcontroller is a $10 \mathrm{k}$ resistor used to limit the flow of current in the oscillatory circuit of the microcontroller.

6. It is powered using a $5 \mathrm{~V}$ phone charger and a USB cable.

The software used for this paper is Proteus software and Mikro C software, see appendix A for circuit codes. The Proteus Design Suite is an Electronic Design Automation (EDA) tool including schematic capture, simulation and PCB Layout modules. "It is developed in Yorkshire, 
England by Lab center Electronics Ltd with offices in North America and several overseas sales channels. The software runs on the Windows operating system and is available in English, French, Spanish and Chinese languages" (10). "The MikroC PRO for PIC is a full-featured ANSI C compiler for PIC devices from Microchip ${ }^{\circledR}$. It is the best solution for developing code for PIC devices. It features intuitive IDE, powerful compiler with advanced optimizations, lots of hardware and software libraries, and additional tools that will help you in your work (11)”.

\section{B. Simulated Circuit Diagrams}

This section consists of the circuit diagrams and PCB layout of the simulated circuit.

\section{Seven Segment to Display Number 0-9}

Figure 4 and 5 below shows the Schematic diagram and PCB Layout of the circuit respectively.

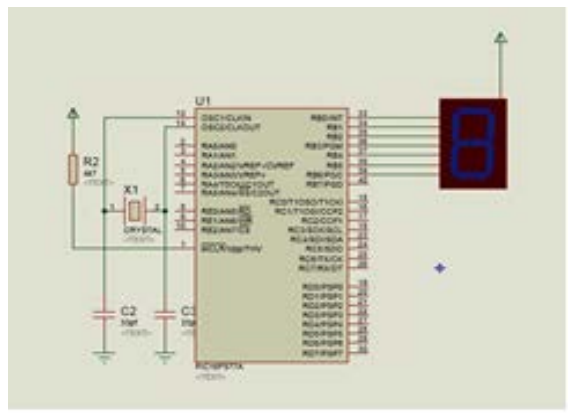

Fig. 37 Segment 0-9 Schematic Diagram

This circuit uses a microcontroller which is powered by a 5V DC input from a charger. The port B of the microcontroller is used to send output to a Common Anode 7 Segment display LED. See appendix A below for the code.

\section{Seven Segment Counter to Count 0-99}

Figure 6 shows the circuit diagram and pcb layout respectively.

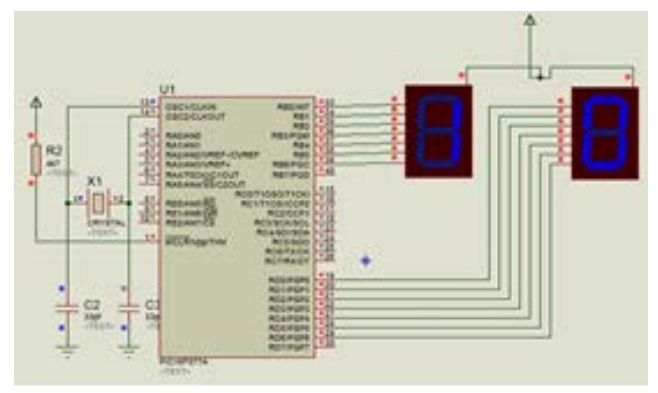

Fig. 4 The Schematic Diagram

This circuit is powered by a 5V DC input from a charger. Connected to the $11^{\text {th }}$ and $12^{\text {th }}$ pin of the microcontroller, the oscillatory circuit too is connected to regulate the clock impulse of the microcontroller. Port B and Port D of the microcontroller was used to send output to a Common Anode 7 Segment display LED. See appendix A for the codes.

\section{Matrix Led to Display A-Z}

A Matrix LED is an arrangement of LED in rows and columns with special array arrangement. For this paper a 5X7 Matrix LED was used to display alphabetical output from letter $\mathrm{A}-\mathrm{Z}$.

Port C and D of the microcontroller were used to connect the rows and columns respectively. A micro $C$ program was also written to display the expected output using PIC18F452 and a NAND gate IC to invert the output. Figure 8 and 9 below shows the circuit diagram and PCB layout of the simulation respectively. See code on appendix A.

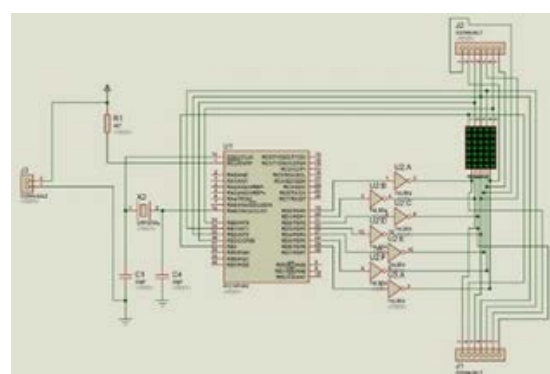

Fig. 5 Schematic Diagram of a Matrix 5X7 to display A-Z

\section{Digital Thermometer LM35}

Thermometer is a device that is used to measure the amount of coldness or hotness of a body, environment or atmosphere. Here, a digital thermometer is designed using microcontroller PIC 16F877A. The main components in the paper are

1. LM35: for sensing temperature,

2. Microcontroller: to receive input and give output and

3. LCD: to display to the user the temperature reading of the LM35

This paper is powered using USB 5V DC Supply, the circuit diagram and PCB of the paper is shown in the Figure 10 and 11 below.

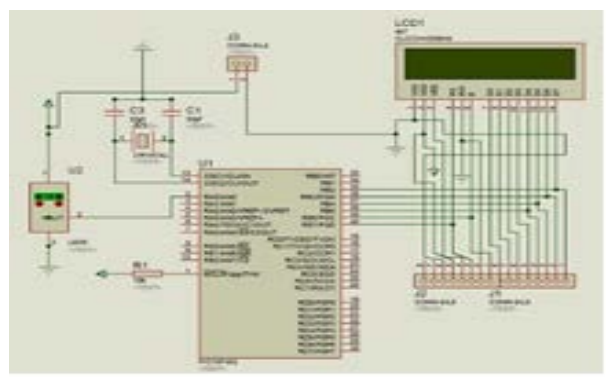

Fig. 6 The Schematic Diagram 


\section{Ten Points of Light Controlled by Motion Sensor}

This is a ten light point controlled by the trigger of Motion Sensor. This work uses a PIR Motion Sensor, a PIC16F877A Microcontroller, and an oscillatory circuit, relay network for lamp switching and header pins. Figure 12 shows a miniature ten point of light and one motion sensor used for circuit simulation while Figure 13 shows the circuit diagram and PCB layout of the paper. See code in Appendix A.

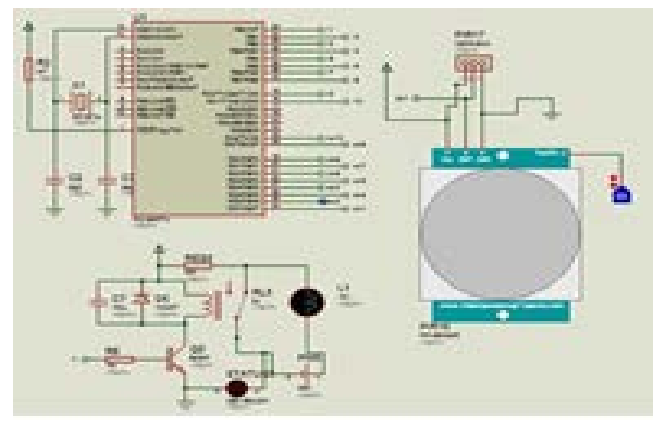

Fig. 7 A Miniature Ten Point of Light Controlled by a Motion Sensor

\section{RESULT, TESTING AND DISCUSSION}

The construction of the paper involved the process of designing, coding and simulation. Then after confirming the working simulation, the PCB was produced and it was constructed and tested, the pictures of the paper is as shown below.

\section{A. Segment to Display 0-9}

The picture in Figure 15, 16 and 17 is 7 Segment to display Number 0-9, during construction and after packaging. The arrangement of the seven segment pins ranges from $\mathrm{A}$, B, C, D, E, F, G and DP.

In constructing this paper is very essential to arrange the pins on the board according to the arrangement of the pins of the original 7 SEGMENT device.

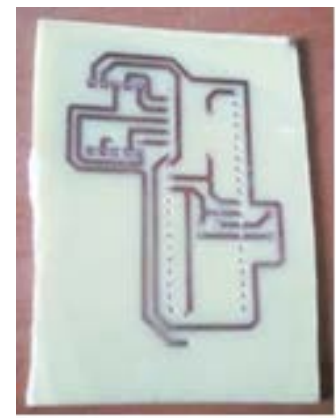

Fig. 8 The Circuit after Drilling, Awaiting Components and Soldering

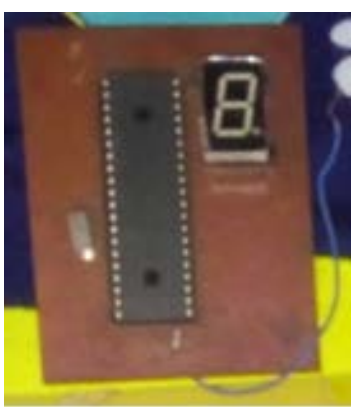

Fig. 9 After Arranging the Components and Soldering

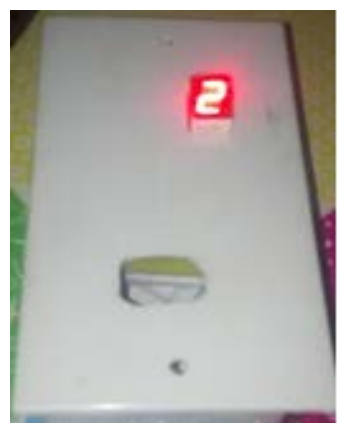

Fig. 10 After Packaging the Completed Working Circuits

\section{B.7 Segment to Display 0-99}

The picture in Figure 18, 19 and 20 is 7 Segment to display Number 0-9, during construction and after packaging.

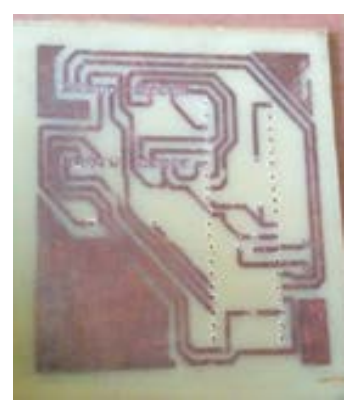

Fig. 11 Before the Soldering, after the Drilling of the Circuits

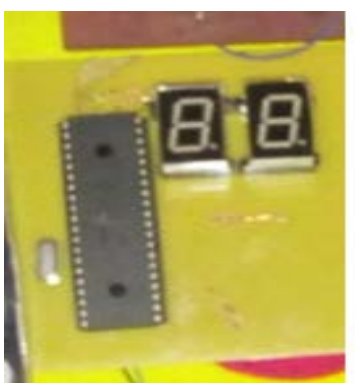

Fig. 12 After Arranging the Components and Soldering 


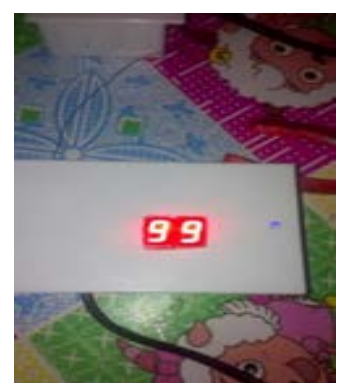

Fig. 13 After the Completion of the Project

\section{Digital Thermometer using LM35}

The picture in Figure 21, 22 and 23 is 7 Segment to display Number 0-9, during construction and after packaging. The LM35 is a very sensitive sensor; it changes the temperature almost immediately.

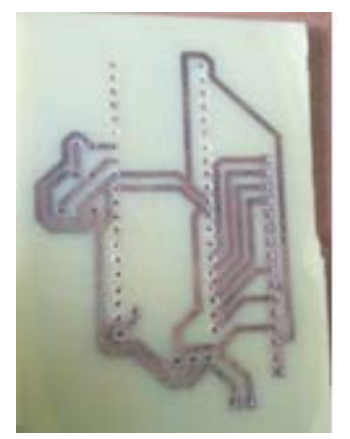

Fig. 14 The Printed Board after Soldering

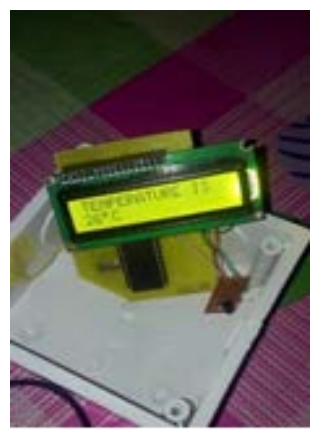

Fig. 15 After the Arrangement and Soldering

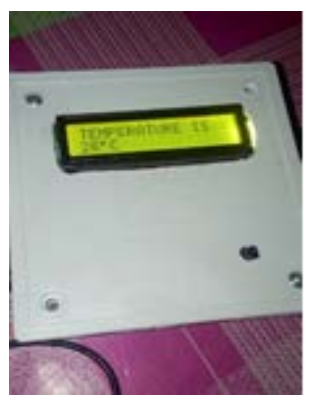

Fig. 16 After Packaging the Project

\section{Matrix Led To Display A-Z}

The picture in Figure 24 and 25 shows the PCB after transferring the circuits and after soldering the components on the board.

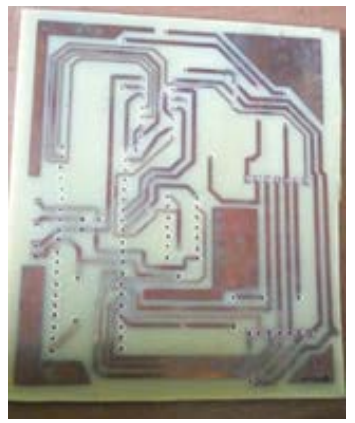

Fig. 17 After drilling before arranging the components

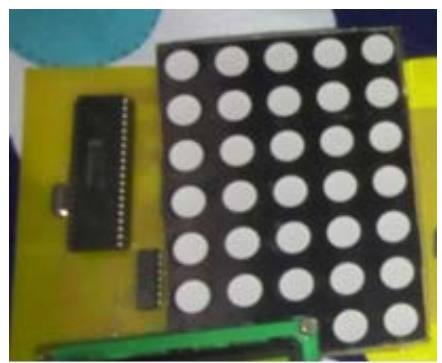

Fig. 18 After arranging the Components on the Board and Soldering

\section{E. 10 Points of Light Controlled by a Motion Sensor}

The picture in Figure 26 and 27 shows the PCB after transferring the circuits and after soldering the components on the board.

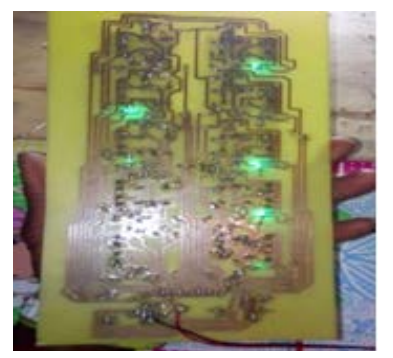

Fig. 19 The PCB Layout of the Circuit

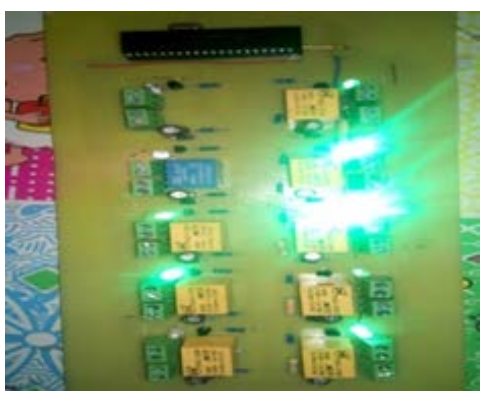

Fig. 20 The Top Layout of the Circuit 


\section{F. Testing}

\subsection{Segment to Display 0-9}

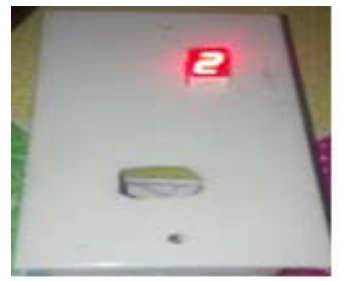

Fig. 21 The finished picture of 7 segments to display 0-9

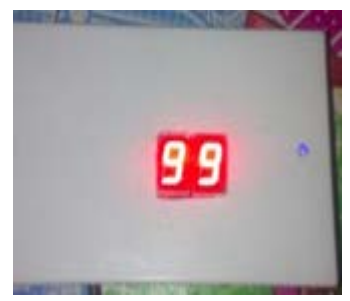

Fig. 22 The finished picture of 7 segments to display 0-99

\subsection{Segment to Display 0-99}

This completed package of the paper is shown on Figure 25 above, it is more useful for training students and for practice.

\section{Digital Thermometer using LM35}

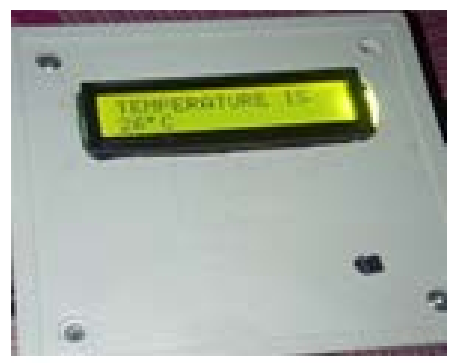

Fig. 23 The finished picture of digital thermometer using LM 35

The finished work use a 5V DC supply from a USB cable to power, it is useful for home, office or industrial use.

\section{G. Discussion}

\section{Economic Benefits of Seven Segments to Display 0-9}

In terms of academics, it is a design that would enhance study and use of Mikro C programming and Circuit Simulation.

In terms of Reliability, it is very reliable, once it is well designed and constructed, it is very reliable.

In terms of human management the touch switch can be used to save time and reduce stress, it can also serve as a good source for home automation gadget or for exhibition. In terms of maintenance, it is easily maintained and saves energy too.

\section{Advantages of Seven Segment to Display 0-9}

1. It would increase the use of $C$ language programming among students.

2. It would help student become efficient in working as an Engineer.

3. It would help lectures reduce stress of teaching the practical aspect of engineering.

\section{H. Area of Improvement}

It can be further improved using a development board.

\section{Economic Benefits of Seven Segments to Display 0-99}

In terms of academics, it is paper that would enhance study and use of Mikro C programming and Circuit Simulation. In terms of Reliability, it is very reliable, once it is well designed and constructed. In terms of maintenance, it is easily maintained and saves energy too.

\section{Advantages of Seven segments to Display 0-99}

1. It would increase the use of $C$ language programming among students.

2. It would help student become efficient in working as an Engineer.

3. It can serve as a very vital equipment of learning for children under age 2-4.

4. It would help lectures reduce stress of teaching the practical aspect of engineering.

\section{Economic Benefits of Digital Thermometer using LM35}

In terms of academics, it is paper that would enhance study and use of Mikro C programming and Circuit Simulation. In terms of Reliability, it is very reliable, once it is well designed and constructed In terms of maintenance, it is easily maintained and saves energy too.

\section{Advantages of Digital Thermometer using LM35}

1. It would increase the use of $\mathrm{C}$ language programming among students.

2. It can be used for demonstration.

3. It can be used to detect atmospheric temperature.

4. It would help lectures reduce stress of teaching the practical aspect of engineering .

\section{Economic Benefits of Matrix Led to Display A-Z}

In terms of academics, it is paper that would enhance study and use of Mikro C programming and Circuit Simulation. 
In terms of Reliability, it is very reliable, once it is well designed and constructed. In terms of human management, it serves as a good source for learning. In terms of maintenance, it is easily maintained and saves energy too.

\section{Advantages of Matrix Led to Display A-Z}

1. It would increase the use of $C$ language programming among students.

2. It can also be used for advertisement depend on the construction.

3. It can serve as a very vital gadget for learning basically for kids between ages 2-4.

4. It would help lectures reduce stress of teaching the practical aspect of engineering.

\section{Economic Benefits 10 Points of Light Controlled by a Motion Sensor}

In terms of academics, it is paper that would enhance study and use of Mikro C programming and Circuit Simulation.

In terms of Reliability, it is very reliable, once it is well designed and constructed. In terms of human management, it can be used to save time and reduce stress; it can also serve as a good source for home automation gadget. In terms of maintenance, it is easily maintained and saves energy too.

Advantages of 10 Points of Light Control by a Motion Sensor

1. It would increase the use of $C$ language programming among students.

2. It can serve as a quickly way of powering house or company.
3. It can serve as a very vital gadget for home automation.

4. It would help lectures reduce stress of teaching the practical aspect of engineering.

\section{CONCLUSION}

The design and construction of PCB of circuits is a good way of analyzing circuits and training students because it makes work neat and saves cost of purchasing components all the time for practical's in schools. While making a PCB prototype, it is important to follow the rules right from photoengraving to testing. Any slight mistake can make the circuit board useless. In case of mass production, PCB prototype is a must. The presented hand-on circuits have proved to be instructive to trainees. Due to the fact that the design of PCB circuits make work neat and save cost, it is therefore recommended in all electronic circuit design but all circuit boards must go through intensive testing and quality control during each step of the manufacturing process.

\section{REFERENCES}

[1] Williams, K. Jan, PCB Material Selection Guide, 2017.

[2] Ghetty and Jame, "PCB Material”, Journal for Material Science and Engineering, 2015.

[3] James and Mike, "РCB History”, Journal for Printed Circuit Board, 2015.

[4] Jack and willams, Important of PCB, 2015.

[5] PCB Material Selection Guid, 2016.

[6] Frank and Hissen, Simulation History, 2012.

[7] Comsol and Satan, 'Electroplating Simulation”, Journal of Printed Circuit Board Design, 15 Dec. 2015.

[8] Tarun and Agarwal, "Types of Printed Circuit Board", 2015.

[9] Malcolm and Wagner, "The Importance of PCB to Electronics", IEE Journal of Electronics, 2015.

[10] Washton and Clara, Brief on Proteus, 2014.

[11] MikroElektronika, History on Mikro C, 2016. 